\title{
Nursing decision support system: application in electronic health records ${ }^{\dagger}$
}

Mi-Zhi Wua, Hong-Ying Pan ${ }^{\mathrm{b}, *}$, Zhen Wang ${ }^{\mathrm{c}}$

${ }^{a}$ School of Nursing Sciences, Huzhou University, Huzhou, Zhejiang 313000, China

${ }^{b}$ Nursing Department of Sir Run Run Shaw Hospital, Medical School Zhejiang University, Hangzhou, Zhejiang 310017, China

' School of Medicine, East Campus of Huzhou University, Huzhou, Zhejiang 313000, China

Received: 22 October 2019; Accepted: 21 November 2019; Published: 20 September 2020

\begin{abstract}
The clinical decision support system makes electronic health records (EHRs) structured, intelligent, and knowledgeable. The nursing decision support system (NDSS) is based on clinical nursing guidelines and nursing process to provide intelligent suggestions and reminders. The impact on nurses' work is mainly in shortening the recording time, improving the quality of nursing diagnosis, reducing the incidence of nursing risk events, and so on. However, there is no authoritative standard for the NDSS at home and abroad. This review introduces development and challenges of EHRs and recommends the application of the NDSS in EHRs, namely the nursing assessment decision support system, the nursing diagnostic decision support system, and the nursing care planning decision support system (including nursing intervene), hoping to provide a new thought and method to structure impeccable EHRs.
\end{abstract}

Keywords: electronic health records $\bullet$ decision support systems $\bullet$ clinical $\bullet$ nursing process $\bullet$ review

(c) Shanxi Medical Periodical Press.

\section{Introduction}

Electronic health records (EHRs) are defined as records of data on individuals, usually located on databases or similar digital media, which can be used by health care clinicians and nurses during an individual's hospitalization. ${ }^{1}$ The nursing decision support system
(NDSS) established nursing knowledge base and method base, then compiled logical reasoning rules, and finally realized the integration with EHRs. ${ }^{2}$ To elaborate, NDSS is developed within the framework of EHRs, where the patient information is matched to the content of the computer knowledge base, aiming to calculate a feasible individualized nursing care plan

How to cite this article: Wu MZ, Pan HY, Wang Z. Nursing decision support system: application in electronic health records. Front Nurs. 2020;3:185-190.

$\dagger$ This project was supported by the Development and application of nursing decision support system based on artificial intelligence (No. 2019ZD006). 
for patients and optimize the nursing decision. ${ }^{2,3}$ Most importantly, NDSS has a human-machine interaction mechanism to complete the patient data input and then generate decision support results to users. ${ }^{4}$ Furthermore, NDSS features an extensive assortment of functions that can contribute to the decision-making in EHRs such as patient data reports, reminders and alerts, clinical guidelines, diagnostic support, and tools for clinical workflow.

Nursing documents in EHRs are crucial to the safety of patient care. Compared with paper nursing records, EHRs lack the framework of nursing process which endangers the continuity of care delivery. ${ }^{5}$ The record of patient care is an explicit goal in which the model of the nursing process is concretized. Another outstanding form of action is the analysis of the content of nursing, and based on this framework the needs of an individual patient can be identified and assessed. ${ }^{6}$ Therefore, this review describes the development, the challenge of EHRs, and the application of NDSS in EHRs based on nursing process, and, in particular, it includes an illustration from the perspectives of nursing assessment, nursing diagnosis, and nursing care plan.

\section{The development and challenges of EHRs}

In recent years, EHRs have become an indispensable tool for medical staff. New computer technology developed in the 1960s and 1970s has laid the foundation for the development of EHRs. ${ }^{7}$ Since the 1990s, EHRs have been introduced to an increasing number of hospitals globally. Compared with the traditional paper medium, EHRs have many potential benefits, such as saving the costs, minimizing the errors, and improving the efficiency achieved through utilizing the real-time data. Due to these advantages, the American Recovery and Reinvestment Act was passed by the US Government in 2009, which mandated that all health care organizations should adopt a certified EHRs system by $2015 .{ }^{8}$ Gradually, EHRs can be used meaningfully and have been brought into focus.

One of the peculiarities of EHRs is the implemented clinical decision support system (CDSS). CDSS applications continue to be promoted as one of the key functions of EHRs because it is the most direct way to integrate with daily workflow once implemented in EHRs. ${ }^{9}$ Modern patient care relies on many different medical devices to monitor patients' vital signs, inject drugs, and provide life-maintaining support. These medical devices include ventilators, infusion pumps, bedside and portable monitors, and other data through custom interfaces. ${ }^{10}$ These data are not only valuable for integrating with patients' EHRs, but also necessary for CDSS applications to help prevent patients from exacerbation. In this way, patient data can be acquired directly based on EHRs, and they are closely combined with a medical workflow to provide real-time on-site decision support for diagnosis and treatment activities and reduce medical errors in medical decision-making. However, the majority of EHRs currently remain a hybrid collection of computerized and paper data. ${ }^{7}$ On the other hand, the enormous amount of patient data makes it difficult, if not impossible, for nurses to effectively process and integrate into patient nursing decisions. ${ }^{11}$ The study by Ozkaynak et al. ${ }^{12}$ demonstrated that nurses can face challenges when using EHRs without nursing process framework that can threaten the quality and safety of care. Kossman et al. ${ }^{13}$ claimed that $73 \%$ of nurses often spend at least half of their work time using EHRs and agree that EHRs enable them to provide safer care but decreased quality of care. In addition, due to the increased workload, some nurses and other medical staffs are highly resistant to EHRs changes. ${ }^{8}$ NDSS connects health observations with the knowledge to help clinical nurses make decisions and improve the quality of care using accurate and structured clinical information provided in EHRs. ${ }^{9}$ Relatively speaking, most EHRs have no access to the NDSS. ${ }^{14}$ Therefore, this article chooses to analyze the application of NDSS based on EHRs.

\section{The application of NDSS in EHRs}

The highest functional level of EHRs is NDSS and process automation which are expected to enhance patient safety and the quality of nursing. ${ }^{15}$ Moreover, the nursing process is a basic cognitive activity that requires critical and creative thinking in care delivery. ${ }^{6}$ It is also a chart format that records all nursing activities and patient outcomes. Hence, we introduce the role of NDSS in EHRs according to the nursing process.

\subsection{The effect of NDSS in nursing assessment}

Nursing assessment is the first step in the nursing process and it is the basis to determine the signs and symptoms that will later support the nursing diagnosis. ${ }^{16}$ In clinical nursing work, the patient's daily nursing assessment work has a large amount of data with complicated content, ${ }^{11}$ which are difficult for nurses to identify, control, and utilize the data collection, or evaluate the results. Ozkaynak et al. ${ }^{12}$ found that nurses face challenges in nursing assessment when using EHRs that can threaten quality and safety of care. The time to record in EHRs 
is estimated to be $4 \mathrm{~h} /$ day. ${ }^{17}$ Furthermore, additional manual data reduce the accuracy of the assessment. ${ }^{18}$ Also, physiological monitors, ventilators, vital signs, anesthesia machines, and other immediate care devices are rarely fully integrated with EHRs, and as a result, nurses are required to manually enter electronic device data into EHRs. ${ }^{19}$ This means that nurses need assistance to become proficient in nursing assessment due to the difficulty of simultaneously processing a large set of information.

EHRs provide a complete set of patient medical data within a health care facility ${ }^{9}$ which provides the most critical condition for EHRs to integrate NDSS modules. Although structured nursing assessment forms based on nursing models are often used in clinical nursing practice, the collected data are not usually translated into the nursing diagnosis. At this point, the nursing assessment decision support system (NADSS) can extract nursing records and other clinical records during hospitalization to supplement structured vital sign data. ${ }^{20}$ Then, the properties of EHR along with the inclusion of diverse aspects of patients' health-related information of the system capturing or the nurse filling that constructs automated risk assessment systems for providing nursing decision supports. ${ }^{21}$ Additionally, NADSS contains a variety of alert functions, such as reminders of outliers in the nursing assessment, reminders of missing entries, and warning of high-risk values.

Bouyer et al. ${ }^{22}$ applied the NADSS in the operating room to deal with nursing records, which assists to increase the intervention measures for peripheral nerve injury, and reduced the incidence of peripheral nerve injury. Similarly, Fan and $\mathrm{Shi}^{23}$ have integrated the NDSS with man-machine dialogue function into the structured EHR and implanted the clinical application module of nursing assessment. Likewise, Luo et al. ${ }^{24}$ used the NDSS in pressure ulcer assessment, application, and consultation, grasped the opportunity of pressure ulcer assessment, and timely prevented and effectively reduced the incidence of pressure ulcers. Hence, inserting the NDSS into EHRs facilitates the integrity of patient data, capturing automatically the existing patient data, reducing nurses' manual judgment errors, and improving the accuracy of nursing assessment.

\subsection{The effect of NDSS in nursing diagnostic}

The nursing diagnosis is the core element of the nursing process because it is the basis for selecting effective nursing interventions. ${ }^{25}$ However, nurses often need to make quick decisions with incomplete diagnostic information especially in time-critical situations. According to the study by King et al., ${ }^{26}$ nurses did not consistently document patient nursing diagnoses, ignoring the holistic nursing care needs of some of their patients. Martin has reported that in her study, only $15 \%$ of nurses are confident regarding a patient's nursing diagnosis. ${ }^{27}$ According to these nurses, the barriers to use a nursing diagnosis include a lack of time, acceptance of the nursing diagnosis, and knowledge about nursing diagnosis. Importantly, NDSS can help nurses solve these two problems-improving nursing diagnosis expertise and determining the appropriate nursing diagnosis. Nursing diagnostic decision support system (NDDSS) is primarily based on evidencebased guidelines for nursing diagnosis database. The diagnostic decision support system (DDSS) is embedded into EHRs, which provides a set of questionnaires for registered nurses to guide them in preparing documents for nursing assessment. ${ }^{28}$ The questionnaire has a drop-down menu with answers. With these answers, the system is capable of calculating probabilities for defining characteristics (DC) and then presenting them to the nurse. The nurse's response thereby is to choose the most important DC. The system again uses the nurse's input and calculates a set of nursing diagnoses (Dx) that best fit the clinical scenario. In special cases, the response during nursing assessment may trigger Dxs instead of DCs. The algorithm is based on the international classification of nursing diagnosis (NANDA-I) and is promoted worldwide. ${ }^{28}$ Therefore, the system can influence the way nurses think about priorities and patient care.

EHRs provide nurses with more competent management of care, in terms of supporting decisionmaking. Cho et al. ${ }^{29}$ have developed NDSS that is data-driven for daily work activities to facilitate the precise and context-sensitive use of the information implied in nursing diagnoses. Liao et al. ${ }^{30}$ explored the application of artificial intelligence in nursing diagnosis by using tools, such as back propagation neural network, to conduct data mining and statistical analysis. However, at least, there are few studies on the application of DDSS in the field of nursing, and even CDSS fails to support nursing practice in major. Future research must address how, when, and why nurses use DDSS, and what standards must be met for nurses to use DDSS effectively. In addition, the impact of DDSS on patient and quality outcomes needs to be studied.

\subsection{The effect of NDSS in nursing care planning}

Nurses need to combine diagnosis and existing scientific evidence in different clinical environments to make nursing care plans for patients, which is a continuous and complex process. ${ }^{31}$ This process requires the ability of the nurse to obtain the best information, think 
about information, filter information, and make clinical decisions. The nursing care planning decision support system (NCPNSS) reminds health care professionals to implement relevant, appropriate, and specific interventions for high-risk or confirmed patients. ${ }^{32}$ It extracts EHRs information that will allow patient-specific personalization of support, access sources of evidence to support the development of individualized plans of care, and provide an overview and detailed description of evidence in EHRs. ${ }^{33}$ It shows that its effect on patient care decisions is positive. In addition, NDSS is based on knowledge discovery, using data mining of nursing care plans.

Examples show that the system has identified patients with pain trends that match historical data, showing that known interventions are effective for these patients based on data mining results, followed by providing decision support to nurses. ${ }^{34}$ The system uses this information to improve the usefulness of the nursing decision.

At present, the NCPNSS is based on case management. ${ }^{31}$ For example, it clarifies the clinical path of the important daily examination, treatment, health education, and nursing plan from patient admission to discharge. Feng and Chang $^{35}$ have developed NCPNSS that guides nurses in identifying patients pain problems and strengthening nursing and pain management. Bowles et al. ${ }^{36}$ have developed the discharge decision support system that evaluates the discharge requirements of patients, develops nursing care plans for discharged patients, and extends the readmission time. In Taiwan, some scholars ${ }^{37}$ have applied clinical care classification (CCC) and evidencebased practice in EHRs to provide a nursing decision, which can reduce nurses' load of nursing plan. NDSS enables nurses to quickly collect patient information in the decision-making process to draw up a rational nursing care plan for patients and improve the decisionmaking level of nursing staff.

\section{Outlook}

To promote the extensive use of EHRs, many countries around the world have made their attempts. In 2009, the Centers for Medicare and Medicaid Services (CMS) incorporated the decision support system into the construction of EHRs. ${ }^{38}$ Furthermore, in 2017, the 13th Five-Year Plan for the development of population health information clearly proposed to use artificial intelligence technology in the construction of medical decision support system. ${ }^{39}$ As NDSS use increases, it must be implemented in a way that is as efficient as possible. Therefore, this article proposes several requests based on the NDSS development.

\subsection{Structure complete NDSS in EHRs}

According to the nursing process, the establishment of the complete NDSS is conducive to the integrity and accuracy of nursing records in EHRs. Nurses rarely record patient outcomes in EHRs. ${ }^{40}$ Patient outcome is an indicator of patient status before and after nursing intervention. Nursing outcome classification (NOC) can be used to determine whether a patient's condition has changed. ${ }^{41}$ There are three levels of classification of nursing outcomes: improvement, stabilization, and deterioration. NOC has been widely used all over the world. However, its use in each country requires some adjustments, in particular, languages. In the future, it will be interesting to study whether the documentation of nursing will improve as nursing outcome is adopted. Moreover, we can apply the traditional user interface design and research methods (e.g., iteration, focus groups, personal interviews, and availability scenario simulation). ${ }^{42}$ In addition to improving functionality, these methods can also create new features to respond to user recommendations. It improves the integrity and practicability of NDSS.

\subsection{The construction of unified standardized nursing terminology in EHRs}

Standardized nursing terminology (SNT) is an important basis for the development of nursing informatization. ${ }^{2}$ SNT is not only used to define care knowledge sorted out in categories, but it is also a prerequisite for building EHRs and implementing electronic health care strategies. ${ }^{14}$ SNT is considered to be the basis for decision support systems that must be able to "exchange data on an international basis and compile data for research and practice." It is essential for data retrieval, statistical analysis, and benchmarking, ensuring clearer, more comprehensive, and faster data entry and retrieval. Importantly, EHRs represent the use of SNT's advanced care procedures, as EHR itself does not guarantee the quality of care. ${ }^{14} \mathrm{SNT}$ provides precise titles and codes for the possibility of nursing diagnosis, intervention, and outcome. Coding concepts allow for evaluation. However, SNT has not yet been fully implemented in clinical nursing practice and nor in EHRs. Therefore, NDSS based on SNT is worth further exploration and research.

\subsection{NDSS meets the need of clinical nursing in EHRs}

NDSS should meet the needs of clinical nurses. There are many forms of decision support system for physicians (including drug-drug interactions and drug dosages), but this system is not widely available 
for nurses in the hospital. Further research is needed to determine whether the decision support system will be accepted and incorporated into real-world workflow to optimize the decision-making for nurses. Moreover, Cho et al. ${ }^{25}$ emphasized that the diverse needs of novices and experts should be considered when designing computer-based decision support and other applications. NDSS should assist not only trainee nurses and nursing novices but also specialists in need in practice areas with which they are not familiar. Therefore, the design team should include nursing domain experts, novice nurses, and data visualization engineers who need to cooperate and collaborate to develop the NDSS functionality. ${ }^{42}$ Besides, the goal is to transform complex analytical results from our statistical and data mining processes into clear and concise text, as well as visual and graphical capabilities which quickly and accurately communicate evidence from EHR data to clinical nurses.

\section{Conclusions}

With the advent of EHRs, hospitals began to recognize the advantages of NDSS and promote clinical

\section{References}

1. Tubaishat A. Evaluation of Electronic Health Record Implementation in Hospitals. Comput Inform Nurs. 2017;35:364-372.

2. Liu $X N$, Pan HY. Application progress of nursing decision support system. Chin J Nurs. 2008;53: 735-739 (in Chinese).

3. Harrison RL, Lyerla F. Using nursing clinical decision support systems to achieve meaningful use. Comput Inform Nurs. 2012;30:380-385.

4. $\mathrm{Xu} \mathrm{JL}, \mathrm{Mao} \mathrm{J}$, Li J. Application progress of clinical decision support system in nursing. J Nurs Sci. 2015;30:103-106 (in Chinese).

5. Hediger H, Muller-Staub M, Petry H. Support of the nursing process through electronic nursing documentation systems (UEPD) - Initial validation of an instrument. PFLEGE. 2016;29:125-135.

6. Lauri S. Development of the nursing process through action research. J Adv Nurs. 1982;7:301-307.

7. Evans RS. Electronic health records: then, now, and in the future. Yearb Med Inform. 2016;25 S48-S61.

8. Barrett AK. Electronic Health Record (EHR) organizational change: explaining resistance through profession, organizational experience, and EHR communication quality. Health Commun. 2018;33:496-506. reasoning. ${ }^{40}$ The clinical nursing task is heavy, the patient condition changes quickly, and nurses are requested to quickly come up with the most suitable patient nursing strategy. NDSS can collate patient data from EHRs and consider evidence-based guidelines to detail the differential diagnosis and recommend follow-up interventions to validate the nurse's thought process. It must include research-based assessment cues, predefined nursing diagnoses, and correct associations among diagnosis, intervention, and patient outcomes. Eventually, such a system can improve the quality of care. ${ }^{13}$ However, NDSS is missing in most EHRs, so we still need to strengthen the NDSS construction. With the development of global medical informatization, the development trend of NDSS is unstoppable.

\section{Ethical approval}

Ethical issues are not involved in this article.

\section{Conflicts of interest}

All contributing authors declare no conflicts of interest.

9. Yang HM, Zhou Y, Tian XY. The application of smart health based on EHR and decision support in precision health management. Smart Healthcare. 2016;2:19-22 (in Chinese).

10. Scott ER, Carlson R, Johnson KV, et al. Enhanced notification of infusion pump programming errors. Stud Health Technol Inform. 2010;160:734-738.

11. Lopez KD, Wilkie DJ, Yao Y, et al. Nurses' numeracy and graphical literacy: Informing studies of clinical decision support interfaces. J Nurs Care Qual. 2016;31:124-130.

12. Ozkaynak M, Reeder B, Hoffecker L, et al. Use of electronic health records by nurses for symptom management in inpatient settings: a systematic review. Comput Inform Nurs. 2017;35:465-472.

13. Kossman SP. Perceptions of impact of electronic health records on nurses' work. Stud Health Technol Inform. 2006;122:337-341.

14. Müllerstaub M, Paans W. A standard for nursing process-clinical decision support systems (NP-CDSS). Stud Health Technol Inform. 2015;225:810-811.

15. Rothman B, Leonard JC, Vigoda MM. Future of electronic health records: implications for decision support. Mt Sinai J Med. 2012;79:757-768.

16. Zega M, D'Agostino F, Bowles KH, etal. Development and validation of a computerized assessment form 
to support nursing diagnosis. Int J Nurs Knowl. 2014;25:22-29.

17. Penoyer DA, Cortelyou-Ward KH, Noblin AM, et al. Use of electronic health record documentation by healthcare workers in an acute care hospital system. J Healthc Manag. 2014;59:130-144.

18. Chiang J, Furler J, Boyle D, et al. Electronic clinical decision support tool for the evaluation of cardiovascular risk in general practice: a pilot study. Aust Fam Physician. 2017;46:764-768.

19. Ancker JS, Edwards A, Nosal S, et al. Effects of workload, work complexity, and repeated alerts on alert fatigue in a clinical decision support system. BMC Med Inform Decis Mak. 2017;17:36-39.

20. Patterson OV, Jones M, Yao Y, et al. Extraction of vital signs from clinical notes. Stud Health Technol Inform. 2015;216:1035.

21. Hung C, Lin C, Lan T, et al. Development of an intelligent decision support system for ischemic stroke risk assessment in a population-based electronic health record database. PLoS One. 2019;14:e0213007.

22. Bouyer-Ferullo S, Androwich IM, Dykes PC. Clinical decision support and perioperative peripheral nerve injury: a quality improvement project. Comput Inform Nurs. 2015;33:238-248.

23. Fan Y, Shi QX. Application of clinical decision support system in nursing evaluation. Chin Health Qual Manage. 2018;25:62-64 (in Chinese).

24. Luo ZJ, Long $\mathrm{Y}, \mathrm{Hu} \mathrm{XH}$, et al. Application of informatization in monitoring of sensitivity quality index of pressure sores. Chin Health Qual Manage. 2017;24:55-58 (in Chinese).

25. Cho IS, Staggers N, Park I. Nurses' responses to differing amounts and information content in a diagnostic computer-based decision support application. Comput Inform Nurs. 2010;28:95-102.

26. King VM, Chard ME, Elliot T. Utilization of nursing diagnosis in three Australian hospitals. Nurs Diagn. 1997;8:99-109.

27. Martin K. Nurse practitioners' use of nursing diagnosis. Nurs Diagn. 1995;6:9-15.

28. Ciqueto Peres HH, De Almeida Lopes Monteiro Da Cruz, Diná, Tellez M, et al. Usability testing of PROCEnf-USP: a clinical decision support system. Stud Health Technol Inform. 2015;216:247-250.

29. Cho I, Kim J, Kim JH, et al. Design and implementation of a standards-based interoperable clinical decision support architecture in the context of the Korean EHR. Int J Med Inform. 2010;79:611-622.

30. Liao P, Hsu P, Chu W, et al. Applying artificial intelligence technology to support decision-making in nursing: a case study in Taiwan. Health Informatics J. 2015;21:137.

31. Hao AT, Hsu CY, Li FH, et al. Apply creative thinking of decision support in electrical nursing record. Stud Health Technol Inform. 2006;124:313-319.

32. Wong CM, Wu SY, Ting WH, et al. An electronic nursing patient care plan helps in clinical decision support. Stud Health Technol Inform. 2015;216:945.

33. Demnerfushman D, Seckman C, Fisher C, et al. Continual development of a personalized decision support system. Stud Health Technol Inform. 2013; 192:175-179.

34. Almasalha F, Xu D, Keenan GM, et al. Data mining nursing care plans of end-of-life patients: a study to improve healthcare decision making. Int J Nurs Knowl. 2013;24:15-24.

35. Feng RC, Chang P. Developing evidence-based care standards and a decision-making support system for pain management. Stud Health Technol Inform. 2016;225:887-888.

36. Bowles $\mathrm{KH}$, Hanlon A, Holland D, et al. Impact of discharge planning decision support on time to readmission among older adult medical patients. Prof Case Manag. 2014;19:29-38.

37. Huang $\mathrm{HL}$, Lee $\mathrm{CY}$, Chuang PL, et al. Using mode of clinic care classification in clinical nursing information system. Stud Health Technol Inform. 2016;225:1064-1065.

38. Carley S. The American Recovery and Reinvestment Act of 2009: what have we learned?: the American recovery and reinvestment act. Rev Policy Res. 2016;33:119-123.

39. Chinese Journal of Health Informatics and Management. Introduction of "thirteenth five-year plan of national population health information development plan". Chin J Health Inf Manage. 2017;14:13-14 (in Chinese).

40. Minna M, Kaija S, Merja M. Nursing audit as a method for developing nursing care and ensuring patient safety. NI 2012 (2012). 2012;2012:301.

41. Adistya VK, Nurjannah I, Subekti H. The interrater reliability of nursing outcome classification (NOC): "caregiver performance: direct care". Int J Nurs Knowl. 2018;29:192-199.

42. Lopez KD, Febretti A, Stifter J, et al. Toward a more robust and efficient usability testing method of clinical decision support for nurses derived from nursing electronic health record data. Int $J$ Nurs Knowl. 2017;28:211-218.

43. Piscotty R, Kalisch B. Nurses' use of clinical decision support a literature review. Comput Inform Nurs. 2014;32:562-568. 